\title{
The Effectiveness of Functional Electrical Stimulation (FES) in On-Off Mode for Enhancing the Cycling Performance of Team Phoenix at 2016 Cybathlon
}

Kenry WC Leung (1), Raymond KY Tong (1), Xiaojun Wang (1), Ginny TY Lee, Peter MK Pang (2), HW Wai (2), HC Leung (2)

(1) Department of Biomedical Engineering, The Chinese University of Hong Kong, Hong Kong; (2) Industrial Centre, The Hong Kong Polytechnic University, Hong kong.

This article is distributed under the terms of the Creative Commons Attribution Noncommercial License (CC BY-NC 4.0) which permits any noncommercial use, distribution, and reproduction in any medium, provided the original author $(s)$ and source are credited.

\begin{abstract}
In this study we designed a Functional Electrical Stimulation (FES) trike for a female subject with spinal cord injury to exercise her lower limbs and improve her lower limb muscle condition for attending the 2016 Cybathlon FES bike competition. Our FES pilot was the only female participant, in the FES cycling competition and she rode for Team Phoenix from the Chinese University of Hong Kong. Due to the weakness of muscles in the lower limb of the subject, and due to scoliosis over her thoracolumbar aéra, the mechanical structure of the trike had to be tailor-made to ensure she sat on the bike in a safe and secure position. A six-phase angle-driven stimulation pattern was developed to stimulate quadriceps and hamstrings without gluteus muscles for contraction through four surface electrodes, thereby creating a cycling movement. To improve the cycling endurance and reduce the muscle fatigue, an on-off mode was developed for controlling the stimulation time that allowed the subject to cycle for 20 s, then pause while the trike advanced without stimulation for $5 \mathrm{~s}$, followed by a subsequent $20 \mathrm{sec}$ stimulation, to continue cycling. The pilot participated in the training procedure including training exercise at home, trike fitting in the trike by modifying the mechanical structure, and conducting the cycling exercise for six months. We observed significant improvements in the pilot's lower limb condition. The on-off mode enabled our pilot to extend her cycling endurance effectively, from 1 $\mathrm{min}$ to $2.5 \mathrm{mins}$ and the distance from $62 \mathrm{~m}$ to $100 \mathrm{~m}$. Over the eight minutes time limit, our team successfully finished $100 \mathrm{~m}$ in the Cybathlon FES.
\end{abstract}

Key Words: Functional Electrical Stimulation, Spinal Cord Injury, Cycling, Fatigue

Eur J Transl Myol 27 (4): 302-307

The Cybathlon is a unique competition, which aims to promote the research, development, and implementation of assistive technologies for people with disabilities or physical weakness to compete against each other in six disciplines which include the, Brain-Computer Interface (BCI) Race, Functional Electrical Stimulation (FES) Cycling Race, Powered Arm Prosthesis Race, Powered Leg Prosthesis Race, Powered Exoskeleton Race, and Powered Wheelchair Race.

FES is a technique in which a stimulation device generates electrical pulses to skillfully stimulate the paralyzed muscles for timed contractions by placing electrodes over the skin or implanting them near the muscle motor points. Thus, people with spinal cord injury (SCI) can use an intelligent control device to accomplish certain tasks, such as standing, walking, and cycling. ${ }^{1}$
This study designed a six-phase-angle driven FES trike with a subject-specific control algorithm for the female SCI subject to exercise her lower limb and improve her muscle condition. In additional to improving the cycling endurance and reduced muscle fatigue, the on-off mode was developed to optimize cycling performance for the Cybathlon FES cycling competition in Zurich on October 8th, 2016 with Team Phoenix from the Chinese University of Hong Kong.

\section{FES pilot details}

Our pilot was a 20-year-old female SCI subject with thoracic spinal fracture and she was the only female participant in the FES cycling race during the 2016 Cybathlon (Fig. 1). She was $50 \mathrm{~kg}$ with $155 \mathrm{~cm}$ who suffered a spinal cord decompression in March 2011 and posterior spinal fusion was deformed. She is categorized as an AISA A that has completely loss of motor and 


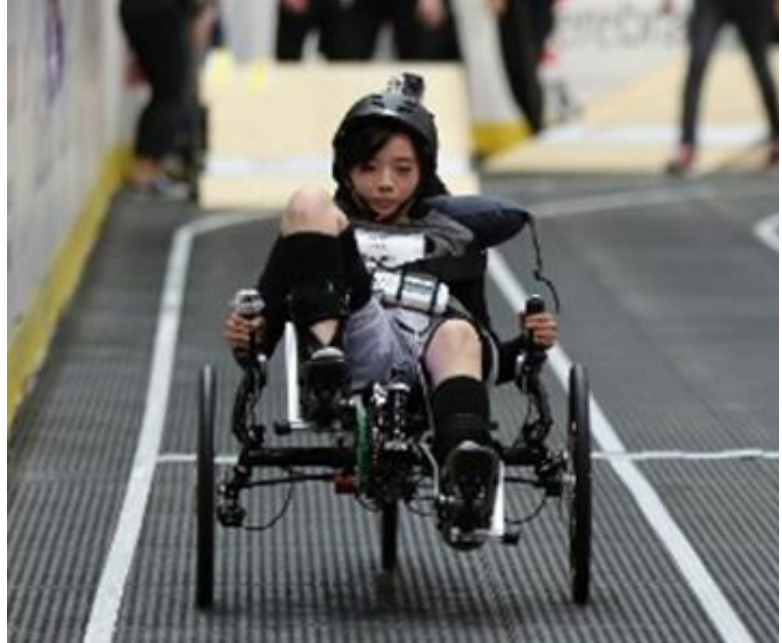

Fig 1. The SCI subject sitting on the modified trike during competition in the Cybathlon in October 2016

sensory functions below the T6-T7 injury level and scoliosis over the thoracolumbar area. Consent was obtained from the subject and the cycling system study was approved by the Joint Chinese University of Hong Kong-New Territories East Cluster (CUHK-NTEC) Clinical Research Ethics Committee (Ref. no: 2016.093-T).

\section{Trike}

Throughout the preparation period before the Cybathlon, a commercial trike (ICE Adventure 26, UK) (Fig. 2) with the following dimensions was used width $82.5 \mathrm{~cm}$, height $80 \mathrm{~cm}$, length $190 \mathrm{~cm}$, and weight $18 \mathrm{~kg}$. The frame of the trike was made of 4130 chromoly steel cruciform and 7005 series aluminum heat treated boom and rear section. For the wheels and tires, Spicer $56 \mathrm{~cm} \mathrm{X} 4 \mathrm{~cm}$ and Tryker Tyre $40.6 \mathrm{~cm} \mathrm{X} 4 \mathrm{~cm}$ were used for the rear and front tires respectively. An encoder was installed and chained to the gear of the crank that was used for obtaining the real-time crankset angle. The fixed 13:22 gear ratio was adopted that allowed the subject to cycle comfortably after our pilot testing with the subject.

\section{Functional Electrical Stimulation (FES) Device}

A modified portable four-channel programmable stimulator (FineCure Easy Walker, P2-9632, China) was integrated into the system and controlled by our designed algorithm. The stimulation parameters adjustable in the frequency range from $1 \mathrm{~Hz}$ to $120 \mathrm{~Hz}$ with the bandwidth between $100 \mu \mathrm{s}$ and $420 \mu \mathrm{s}$. The intensity could be changed for providing a current amplitude from $0 \mathrm{~mA}$ to $100 \mathrm{~mA}$. The electrical pulses generated from the FES device were transmitted to the quadriceps and hamstrings of both legs, using surface electrodes (PALS, Neurostimulation Electrodes $5 \mathrm{~cm} * 9 \mathrm{~cm}$ ) to trigger the muscle contraction.

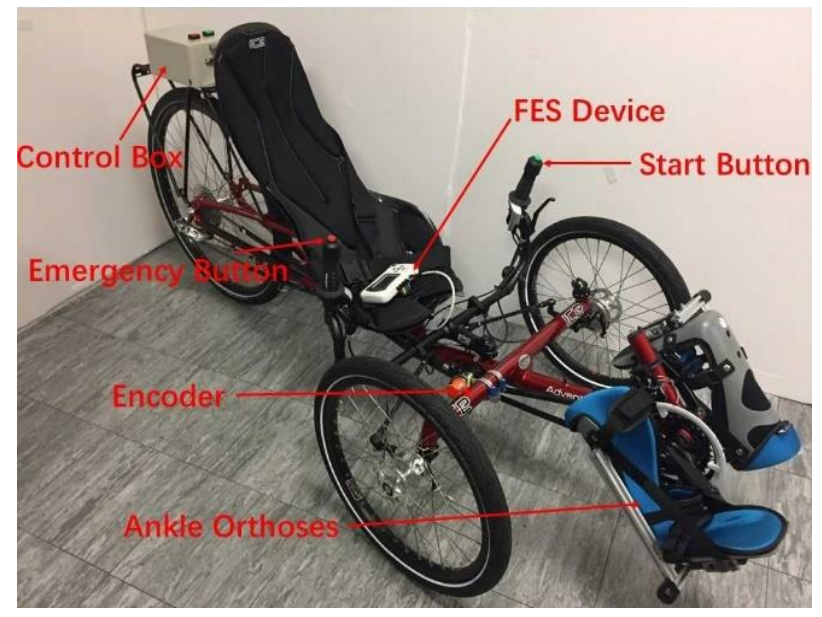

Fig 2. Finalized outlook of the adapted Trike

\section{Challenges}

Because of the physical condition of the subject, the challenges during the development of the FES bike were: 1) a mechanical structure design to fix the position the paralyzed lower limb; 2) a stimulation pattern to generate continuous cycling movement; and 3) an effective cycling mode to improve the endurance and reduce muscle fatigue. We also had to consider an issue related to the pilots comfort and personal needs. For example, the electrode placement, when designing the training protocol initially called for six muscle groups to be stimulated for cycling. However, our pilot had concerns in putting the electrodes on her gluteus muscle due to inconvenient access of this location and the need for help from others all the time. She preferred no electrodes on these muscles. Therefore, an additional challenge was to use only four muscle groups rather than six for stimulation during cycling.

\section{Mechanical Design}

The physical condition of the subject, who has scoliosis over the thoracolumbar area, restricted her from sitting in a straight posture and caused an unbalanced force delivery to the pedals. Therefore, reinforced ankle orthoses (Fig. 3) were designed for maintaining the legs in the sagittal plane in order to optimize the force applied to the pedal. An anatomically contoured shape hard-shell carbon fiber seat with a seatbelt was also used for preventing unwanted body movement of the pilot and maintaining her in a safe and secure position.

\section{Six-Phase-Angle-Driven Stimulation Pattern}

A six-phase-angle-driven stimulation pattern was designed to facilitate the subject cycling on the bike continuously. The initiation of the stimulation pattern was based on the real-time crank angle feedback from the encoder. The targeted muscles were stimulated to complete a sequence of cycling movement with a clockwise direction from $0^{\circ}$, the initial position of the 


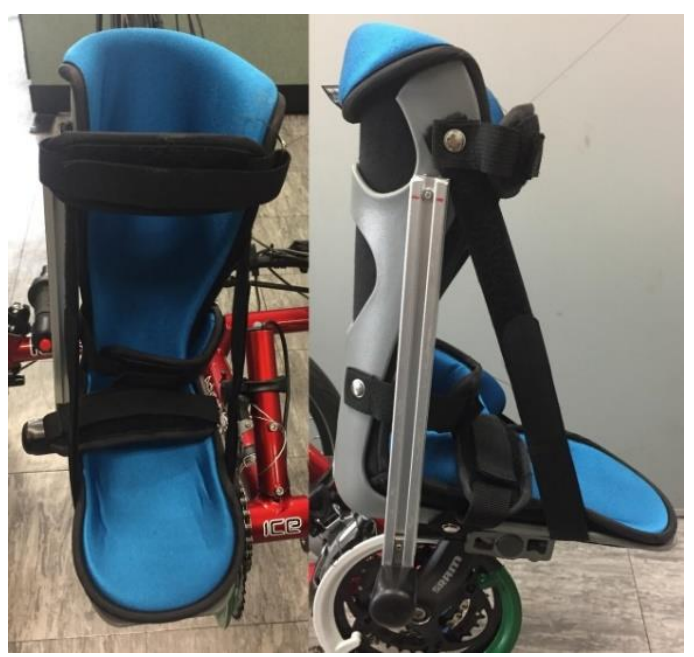

Fig 3. The front view (left) and side view (right) of the reinforced ankle orthoses

crank arm. Fig. 4 showed that the first half of the stimulation pattern was phase 1,2 , and 3 . In phase 1 , the right quadriceps and left hamstrings were stimulated in order to drive the right pedal forward and the left pedal backward at the same time respectively when the crank angle was between $330^{\circ}$ and $60^{\circ}$. After the crank angle was between $60^{\circ}$ and $120^{\circ}$, the right quadriceps were stimulated only. This sequence kept pushing the right pedal forward to generate enough momentum for passing through Phase 3, during which no muscles stimulation was performed (e.g. phase 3 was considered a transition period). The second half of the stimulation pattern from $150^{\circ}$ to $360^{\circ}$ was phase 4,5 , and 6 and is symmetric to the first half of the stimulation pattern, but with left quadriceps and right hamstrings instead.

\section{Cycling Modes}

Two cycling modes, continuous mode ${ }^{2}$, and on-off mode, were designed (Fig. 5) that find which mode was more suitable and effective to extend the cycling endurance and reduce muscle fatigue during cycling. We noticed that the optimal sequence was dependent on the physical condition of the muscles of the subject. For the continuous mode, the stimulation pulses were sent to the targeted muscles repeatedly to maintain a continuous cycling movement until muscle fatigue. For the on-off mode, it was based on a typical cycling pattern from health subjects. The system enabled the subject to cycle for $20 \mathrm{~s}$ with the rest for $5 \mathrm{~s}$ and cycle again. During the rest period, both quadriceps and hamstring had no stimulation and provided a muscle recovery period. Meanwhile, the bike kept advancing sliding freely, and the right leg was positioned at $330^{\circ}$ in phase 1 where was in the ready position to push the pedal for the next stimulation cycle. This provided sufficient recovery to reduce muscle fatigue.

Training Procedure and Trike Development

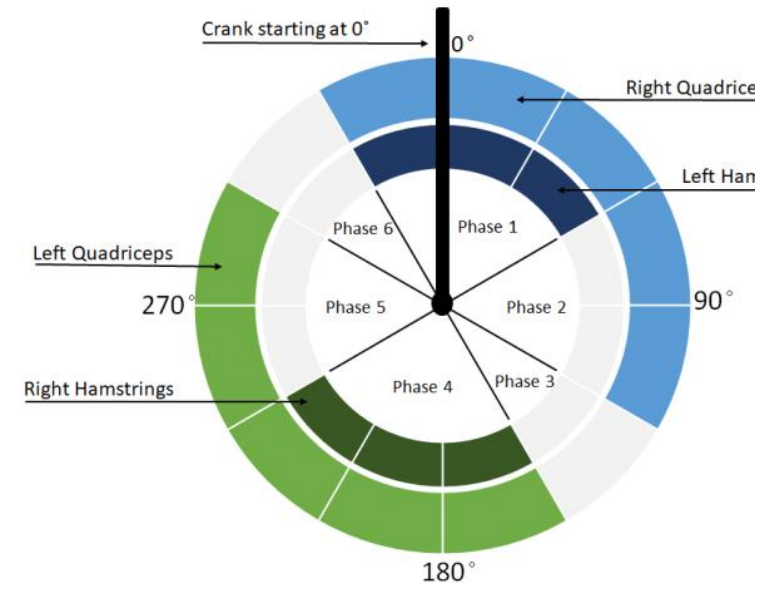

Fig 4. Six-phase-angle-driven stimulation pattern for stimulating the targeted muscles.

The training procedure is presented in time sequence including training at home, trike fitting, and cycling exercise. At the first stage, a training protocol was designed for the subject to conduct the training at home daily for two months, which aimed to improve the muscle condition, such as reduce the edema and muscle atrophy, ${ }^{3-7}$ and increase the muscle strength, ${ }^{8,9}$ of the subject for the cycling exercise. During the training, the right quadriceps and the left hamstrings were grouped as a pair, and the left quadriceps and the right hamstrings were grouped as the other pair. By using the FES device, these muscle groups were stimulated with $50 \mathrm{~Hz}$ stimulation pulses and $360 \mu$ s bandwidth in every $10 \mathrm{~s}$ alternately. The stimulation intensity was adjusted until the subject performed the further extension and flexion of knee for $30^{\circ}$ and $15^{\circ}$ respectively from the initial resting position when the knee was hanging freely at knee angle at $90^{\circ}$ (Fig. 6). These two angles were obtained to ensure sufficient muscle power to facilitate the cycling movement during FES. After training for two months, the muscle condition in both legs was significantly improved, such as larger and longer endurance, to push the pedal forward that enabled the cycling movement with the aid of FES. Meanwhile, our designed six-phase-angle-driven stimulation pattern was developed. During the trike fitting, the trike was placed on a stationary roller, and the subject sat on the trike to cycle several times by using our designed stimulation pattern with the same stimulation intensity as that while training at home. The crankset angle range for pushing forward and pulling backward of pedal were optimized. During this period, the complications caused by scoliosis over the thoracolumbar area provoked an unstable sitting posture. This affected the force applied to the pedal. Therefore, the reinforce ankle orthoses were installed on the trike crank arm to maintain the legs in the sagittal plane and fix the position of shank. An anatomically contoured shape hard-shell carbon fiber seat with a 

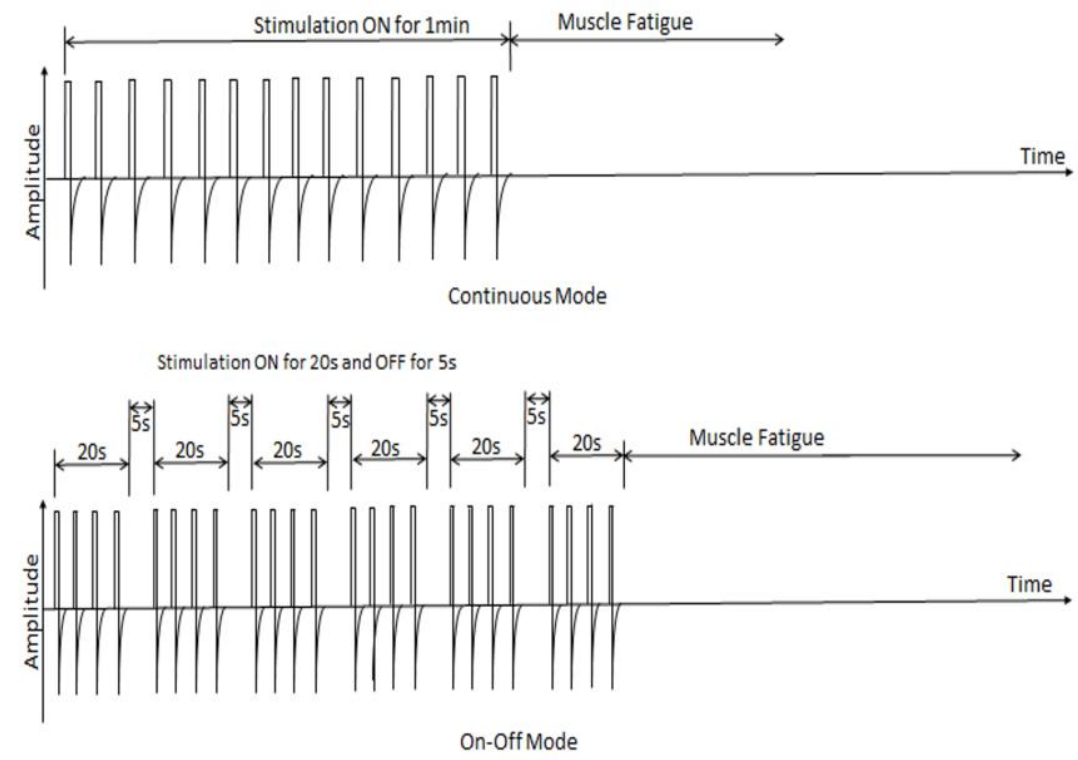

Fig 5. The biphasic waveform stimulation pattern during continuous mode (upper) and on-off mode (lower)

seatbelt was also used for preventing any shift in body posture of the pilot and to keep her hip joint in the proper position. The FES trike was equipped, with a start/stop and emergency buttons on the left and right handles respectively. A rotary encoder for collecting real-time crank angle position with a $200 \mathrm{~Hz}$ sampling frequency used. Two cycling modes were designed for the pilot for cycling exercise. The results showed that the on-off mode showed a better performance, which could effectively improve the cycling endurance and reduce the muscle fatigue. As a result, this mode was selected for the Cybathlon competition.

\section{Results and Discussion}

The pilot training exercises lasted for six months, during which her physical lower limb conditions was observed in terms of reduction of muscle atrophy for the right leg and reducing the edema in the left leg. By comparing both cycling modes, the continuous mode enabled the

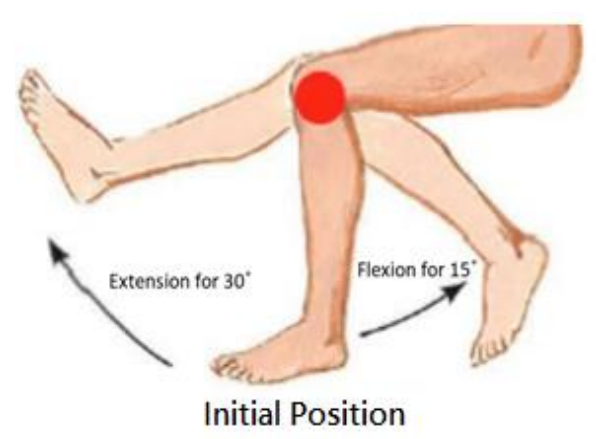

Fig 6. A sketch of the subject performing the extension and flexion of knee during initial setting of the stimulation intensity to muscles subject to cycle around for 1 minute for $62 \mathrm{~m}$ before muscle fatigue. On the other hand, the on-off mode enabled the subject to cycle around 2.5 mins for $100 \mathrm{~m}$ before muscle fatigue. The result showed the on-off mode was more efficient for enhancing the pilot's cycling performance, such as extending the cycling duration, lengthening the travel distance, and reducing muscle fatigue. By comparing with other FES riders in the Cybathlon competition, the short cycling duration and travel distance observed with our pilot were due to the weakness of muscles in the lower limb and rapid muscle fatigue. Performance with our pilot was also limited by only stimulating four major muscle groups bilaterally, i.e. quadriceps and hamstrings, which limited the power generation to drive the trike forward. Therefore, higher stimulation intensity and longer duration were required to each muscle group that caused faster muscle fatigue and affected the competition performance. To improve the performance of cycling, future work would consider six muscle groups to be stimulated. Additionally, more convenient electrode placement for the gluteus muscles would help pilots benefit from the exercise with FES cycling. One possibility would be to integrate the electrodes into spandex cycling shorts.

\section{Concluding Remarks}

Using four muscle groups instead of six, it is feasible to generate cycling movement for SCI persons, but the higher stimulation intensities and longer period were needed which could create faster muscle fatigue. In order to partially overcome this restriction we have developed an On-Off mode that can delay muscle fatigue and extend the cycling duration. Collaboration with other teams present at the first Cybathlon FES Cycling competition and beyond will provide further hints and tips. $^{16-23}$ 


\section{List of acronyms}

AISA A - American Spinal Injury Association (ASIA) Impairment Scale A

FES - Functional Electrical Stimulation

SCI - Spinal Cord Injury

\section{Author's contributions}

All authors contributed equally to this work.

\section{Acknowledgments}

This work was supported in part by an Innovation and Technology Fund (Project No. ITS/276/15) of Hong Kong Special Administrative Region (HKSAR) and Guangzhou Finecure Medical Equipment Company.

\section{Conflict of Interest}

There are no competing interests to report.

\section{Ethical Publication Statement}

Ahthors declared that they have read the Journal's position on issues involved in ethical publication and confirmed that this report is consistent with those guidelines.

\section{Corresponding Author}

Raymond KY Tong

E-mail: kytong@cuhk.edu.hk

\section{E-mails of coauthors}

Kenry WC Leung: wcleung@ee.cuhk.edu.hk Raymond KY Tong: kytong@cuhk.edu.hk Xiaojun Wang: xiaojun27wang@gmail.com Ginny TY Lee: tyleeak@connect.ust.hk HW Wai: honwah.wai@polyu.edu.hk Peter MK Pang: peter.mk.pang@polyu.edu.hk HC Leung: hoi-cheung.leung@ polyu.edu.hk

\section{References}

1. Andrews B, Shippen J, Armengol M, et al. A Design Method for FES Bone Health Therapy in SCI. Eur J Transl Myol 2016;26(4):6419.

2. Hunt KJ, Ferrario C, Grant S, et al. Comparison of stimulation patterns for FES-cycling using measures of oxygen cost and stimulation cost. Med Eng Phys. 2006 Sep;28:710-8.

3. Kaur J. A comprehensive review on metabolic syndrome. Cardiol Res Pract 2014;2014:943162.

4. Davis GM, Hamzaid NA, Fornusek C. Cardiorespiratory, metabolic, and biomechanical responses during functional electrical stimulation leg exercise: health and fitness benefits. Artif Organs 2008;32:625-9.

5. Deley G, Denuziller J, Babault N. Functional electrical stimulation: cardiorespiratory adaptations and applications for training in paraplegia. Sports Med 2015;45:71-82.

6. Mazzoleni S, Stampacchia G, Gerini A, et al. FES-cycling training in spinal cord injured patients.
Conf Proc IEEE Eng Med Biol Soc 2013;2013:5339-41.

7. Fonseca LOD, Bó APL, Guimarães JA2, Gutierrez ME1, Fachin-Martins E2. Cadence Tracking and Disturbance Rejection in Functional Electrical Stimulation Cycling for Paraplegic Subjects: A Case Study. Artif Organs. 2017 Nov;41(11):E185-E195.

8. [8] Pette D, Vrbová G. The Contribution of Neuromuscular Stimulation in Elucidating Muscle Plasticity Revisited. Eur J Transl Myol. 2017 Feb 24;27(1):6368.

9. [9] Stratton K, Faghri PD. Electrically and Hybrid-Induced Muscle Activations: Effects of Muscle Size and Fiber Type. Eur J Transl Myol. 2016 Jul 15;26(3):6163. .

10. Fonseca L, Bo APL, Guimaraes J, et al. Cadence tracking and disturbance rejection in FES cycling for paraplegic subjects: a case study. Eur J Transl Myol 2016;

11. Debelle A, Hermans L, Bosquet M, et al. Soft Encapsulation of Flexible Electrical Stimulation Implant: Challenges and Innovations. Eur J Transl Myol 2016;26(4):6298.

12. Coste CA, Mayr W, Bijak M, et al. FES in Europe and Beyond: Current Translational Research. Eur J Transl Myol 2016;26(4):6369.

13. Valtin M, Kociemba K, Behling $\mathrm{C}$, et al. RehaMovePro: A Versatile Mobile Stimulation System for Transcutaneous FES Applications. Eur J Transl Myol 2016;26(3):6076.

14. Guimarães JA, da Fonseca LO, Dos Santos-Couto-Paz CC, et al. Towards Parameters and Protocols to Recommend FES-Cycling in Cases of Paraplegia: A Preliminary Report. Eur J Transl Myol 2016;26(3):6085.

15. Roth N, Wiener A, Mizrahi J. Methods for Dynamic Characterization of the Major Muscles Activating the Lower Limb Joints in Cycling Motion. Eur J Transl Myol 2014;24(3):3317.

16. Azevedo Coste C, Bergeron V, Berkelman R, et al. Comparison of strategies and performance of functional electrical stimulation cycling in spinal cord injury pilots for competition in the first ever Cybathlon. Eur J Transl Myol 2017;27:251-4.

17. Berkelmans R, Woods B. Strategies and perfor-mances of Functional Electrical Stimulation Cycling using the BerkelBike with Spinal Cord Injury in a competition context (CYBATHLON). Eur J Transl Myol 2017;27:255-8.

18. Laubacher M, Aksöz EA, Bersch I, Hunt KJ. The road to Cybathlon 2016 - Functional electrical stimulation cycling Team IRPT/SPZ. Eur J Transl Myol 2017;27:259-64.

19. Sijobert B, Fattal C, Daubigney A, Azevedo-Coste B. Participation to the first Cybathlon: an overview of the FREEWHEELS team FES-cycling solution. Eur J Transl Myol 2017;27:265-71. 
20. Guimarães JA, Oliveira da Fonseca L, de Sousa AC, et al. FES Bike Race preparation to Cybathlon 2016 by EMA team: a short case report. Eur J Transl Myol 2017;27:272-78.

21. Metani A, Popović-Maneski L, Mateo S, et al. Functional electrical stimulation cycling strategies tested during preparation for the First Cybathlon Competition - a practical report from team ENS de Lyon. Eur J Transl Myol 2017;27:279-88.
22. McDaniel J, Lombardo LM, Foglyano KM, et al. Cycle Training Using Implanted Neural Prostheses: Team Cleveland. Eur J Transl Myol 2017;27:289-94.

23. Arnin J, Yamsa-ard T, Triponyuwasin P, Wongsawat Y. Development of practical functional electrical stimulation cycling systems based on an electromyography study of the Cybathlon 2016. Eur J Transl Myol 2017;27:295-301. 